\title{
Supermassive Hot Jupiters Provide More Favourable Conditions for the Generation of Radio Emission via the Cyclotron Maser Instability - A Case Study based on Tau Bootis b
}

\author{
C. Weber ${ }^{1,2},{ }^{\star}$ N. V. Erkaev, ${ }^{3,4}$, V.A. Ivanov ${ }^{4}$, P. Odert ${ }^{1}$, J.-M. Grießmeier ${ }^{5,6}$, \\ L. Fossati ${ }^{1}$, H. Lammer ${ }^{1}$, and H. O. Rucker ${ }^{7}$ \\ ${ }^{1}$ Space Research Institute, Austrian Academy of Sciences, Schmiedlstr. 6, A-8042, Graz, Austria \\ ${ }^{2}$ Institute of Atmospheric Physics, Czech Academy of Sciences, Prague, Czech Republic (cw@ufa.cas.cz) \\ ${ }^{3}$ Institute of Computational Modelling SB RAS, 660036, Krasnoyarsk, Russian Federation \\ ${ }^{4}$ Siberian Federal University, Krasnoyarsk, Russian Federation \\ ${ }^{5}$ LPC2E - Université d'Orléans/CNRS, France \\ ${ }^{6}$ Station de Radioastronomie de Nançay, Observatoire de Paris, PSL Research University, CNRS/INSU, USR 704 - Univ. Orléans, OSUC, \\ route de Souesmes, 18330 Nançay, France \\ ${ }^{7}$ Comm. Astron., Austrian Academy of Sciences, Graz, Austria
}

Accepted XXX. Received YYY; in original form ZZZ

\begin{abstract}
We investigate under which conditions supermassive hot Jupiters can sustain source regions for radio emission, and whether this emission could propagate to an observer outside the system. We study Tau Bootis b-like planets (a supermassive hot Jupiter with 5.84 Jupiter masses and 1.06 Jupiter radii), but located at different orbital distances (between its actual orbit of $0.046 \mathrm{AU}$ and $0.2 \mathrm{AU}$ ). Due to the strong gravity of such planets and efficient radiative cooling, the upper atmosphere is (almost) hydrostatic and the exobase remains very close to the planet, which makes it a good candidate for radio observations. We expect similar conditions as for Jupiter, i.e. a region between the exobase and the magnetopause that is filled with a lower plasma density compared to cases where the whole magnetosphere cavity is filled with hydrodynamically outward flowing plasma. Thus, unlike classical hot Jupiters like the previously studied planets HD 209458b and HD 189733b, supermassive hot Jupiters should be in general better targets for radio observations.
\end{abstract}

Key words: planets and satellites: aurorae - planets and satellites: magnetic fields - planets and satellites: detection - planets and satellites: atmospheres - planet-star interactions - radio continuum: planetary systems

\section{INTRODUCTION}

It is known that the solar system planets emit low-frequency, coherent, polarized radio emission (e.g. Zarka 1998; Farrell et al. 1999; Ergun et al. 2000; Treumann 2006), where electric fields parallel to the magnetic field accelerate electrons towards the planet into a region of increasing magnetic field. The electrons are partially reflected upwards and some are lost to the planetary atmosphere. The reflected electrons exhibit an unstable distribution, e.g. a loss cone or a horseshoe distribution (e.g. Treumann 2006). The process leading to (exo)planetary radio emission from this electron distribution is the CMI (Cyclotron Maser Instability). It works only if the local electron plasma frequency is smaller than the local electron cyclotron frequency, $\omega_{p}<\omega_{c}$. In particular. it works most efficiently if $\omega_{p} \ll \omega_{c}$.

^ E-mail: cw@ufa.cas.cz, christof.weber@oeaw.ac.at
Thus, the strongest radio emission comes from regions of low plasma density and strong magnetic field.

It is natural to assume that this process is also operating for planets outside the solar system. Various observation campaigns and theoretical studies investigated the possible radio emission from extrasolar planets (see Weber et al. 2017, and references therein). Here, we only highlight two recent theoretical studies by Grießmeier (2018) and Lynch et al. (2018). They extended the studies on detectability of exoplanetary radio emission by Lazio et al. (2004); Grießmeier et al. (2007b) and Grießmeier et al. (2011) to the exoplanet census at the time of their respective studies. Lynch et al. (2018) predict the planets V830 Tau b and $B D+201790 \mathrm{~b}$ to generate observable levels of radio emission in the magnetic and the kinetic energy model, and GJ 876 b only in the kinetic model. Grießmeier (2018) find e.g. Eps Eridani b and Tau Bootis b to be good candidates.

While close-in hot Jupiters have long been considered 
favourable candidates for observing radio emission, it has recently been pointed out by Weber et al. $(2018,2017)$ and DaleyYates \& Stevens $(2017,2018)$ that close-in planets can have hydrodynamically expanded upper atmospheres and ionospheres due to the strong stellar XUV (X-ray and extreme ultraviolet) irradiation. These ionospheres can be very dense, leading to a high local plasma frequency. In some cases, this can lead to quenching of the planetary radio emission in the sense that either no emission can be generated, or that the emission is absorbed in the planetary ionosphere, which extends up to the magnetopause. For a planet of Jupiter's mass or less, one way to prevent this effect would be to look at planets at a larger distance from the star. This, however, weakens the interaction between the star and the planet and the energy input into the planetary magnetosphere, which is usually assumed to be essential for the generation of intense radio emission (a few alternative models do not rely on the proximity of the planet to the host star, see e.g. Grießmeier (2018) for a comparison). Another way to avoid planets with hydrodynamically extended atmospheres and ionospheres would be to study planets around stars with a weaker XUV flux, like the massive A5 star WASP-33 which hosts a hot Jupiter orbiting at a distance of $0.02 \mathrm{AU}$ (e.g. Herrero et al. 2011).

Recently, Hallinan et al. (2015) detected aurorae from a brown dwarf. Since brown dwarfs are very massive their atmospheres should be very compact and they should not have an extended upper atmosphere and ionosphere, but rather a magnetosphere with vast regions of depleted plasma. Thus, they should exhibit source regions where the detected emission is produced and radio waves can freely propagate to an observer on e.g. Earth. Such kind of objects belong to the so-called Ultra Cool Dwarfs and they form a bridge between hot Jupiters and stars (Route \& Wolszczan 2016b; Llama et al. 2018). Hallinan et al. (2008) confirmed that these dwarf stars can have strong dynamos leading to magnetic fields strong enough to generate radio emission via the electron cyclotron maser instability (e.g. Treumann 2006). The authors observed an M-dwarf and an Ldwarf, detecting $100 \%$ circularly polarized and coherent radio emission requiring magnetic fields in the kG-range. These observations confirmed the CMI to be the dominant mechanism for the generation of radio emission in magnetospheres of Ultra Cool Dwarfs (Hallinan et al. 2008). Several other authors reported successfull observations (Berger et al. 2010; Williams et al. 2013; Route \& Wolszczan 2016a,b; Williams et al. 2017).

Since radio emission from Ultra Coold Dwarfs has been already detected and they bridge stars with hot Jupiters, it seems natural to investigate if supermassive hot Jupiters provide better conditions for the CMI than the classical Hot Jupiters studied in Weber et al. (2017). Thus, in this work we are investigating the possible generation of radio emission from Tau Bootis $b$ and the propagation of radio waves in the planetary vicinity. Since the first theoretical studies of exoplanetary radio emission, this planet has frequently been considered as one of the best candidates for radio observations (e.g. Farrell et al. 1999; Grießmeier et al. 2005; Grießmeier et al. 2006a; Grießmeier et al. 2007a,b; Reiners \& Christensen 2010; Grießmeier et al. 2011; Grießmeier 2018). It has been the target of a number of radio observational campaigns (e.g. Bastian et al. 2000; Farrell et al. 2003; Ryabov et al. 2004; Lazio et al. 2004; Shiratori et al. 2006; Winterhalter et al. 2006; Majid et al. 2006; Lazio \& Farrell 2007; Stroe et al. 2012; Hallinan et al. 2013), and more observations of this planet have recently been performed with LOFAR (e.g. Turner et al. 2017).
Here, Tau Bootis b will serve as the archetype of a certain class of planets, i.e. supermassive hot Jupiters. The planet has a minimum mass $(M \sin i)$ of 4.13 Jupiter masses, with an estimated true mass of 5.84 Jupiter masses (www.exoplanet.eu, accessed 2018-06-01). There are currently 92 known exoplanets with more than 2 Jupiter masses and orbital distances of less than $0.1 \mathrm{AU}$.

Twelve of these planets are located at distances $\leqslant 100 \mathrm{pc}$ from Earth. Supermassive hot Jupiters like Tau Bootis b may constitute some of the most promising candidates for radio observations. First, they are likely to have strong magnetic fields. In fact, all magnetic field estimations indicate that massive, gaseous planets should have stronger magnetic fields than less massive Jupiter-like gas giants (Grießmeier et al. 2004; Reiners \& Christensen 2010). This increases the chance to generate radio emission with frequencies above the Earth's ionospheric cutoff of $10 \mathrm{MHz}$ and increases the power that can be received by wide-band radio observations. Secondly, their high masses (and thus larger gravity) lead to more compact upper atmospheres.

For planets more massive than Jupiter, like Tau Bootis b, the planetary gravity keeps the atmosphere strongly bound. In some cases, this will lead to a hydrostatic rather than hydrodynamic upper atmosphere (for example, WASP-18b, is one of these cases; Fossati et al. 2018), so that radio emission could be generated and may escape, a situation which is comparable with the known solar system conditions for e.g. Earth. In other words, a more massive planet maintains hydrostatic conditions closer to its host star than less massive, Jupiter-like planets. This has an added benefit for observational campaigns: close-in planets are easier to study from an observer's point of view, as it is easier to cover one full planetary orbit. In fact, the planetary radio emission is assumed to vary with the planetary orbit, which is one of the ways to discriminate between stellar and planetary emission (see e.g. Grießmeier et al. 2005). The variation is caused by the inhomogeneity of both the planetary magnetic field and the stellar wind (and its magnetic field). For this reason, the stellar rotation has also to be taken into account (Fares et al. 2010), which is particularly important if the stellar rotation and the planetary orbital motion are tidally synchronized (which seems to be the case for Tau Bootis; Donati et al. 2008). Whether the gravity is indeed strong enough to prevent the quenching of planetary radio emission has to be studied on a case-by-case basis; in this follow-up study to Weber et al. (2017), we investigate the case of Tau Bootis $b$.

In the same way as done for the solar system planets, detection of radio emission generated by the CMI yields a direct measurement of the local magnetic field strength in the source regions of the emission, gives the possibility to measure the rotation rate of stars and could be a tool for diagnosing the conditions of the interaction between magnetosphere and ionosphere (Route \& Wolszczan 2016a, and references therein). Furthermore, modelling the dynamic spectrum of radio bursts from Ultra Cool Dwarfs yields information about the topology of their magnetic field (Route \& Wolszczan 2016a, and references therein). Llama et al. (2018) simulated for the first time radio emission from low-mass stars. For this, they used observations of stellar magnetic fields reconstructed via ZDI (Zeeman Doppler Imaging).

Section 2.1 describes briefly the upper atmosphere model used for evaluating the electron density profiles shown in Section 3 and shows the planetary and stellar parameters. This section also describes the upper atmosphere structure of Tau Bootis b at different orbit locations. The estimation of the magnetopause standoff distance as well as the stellar wind parameters are shown in Section 
2.2. This section also shows a comparison of standoff distance and exobase level. Section 3 addresses the results for the plasma environment and the corresponding plasma and cyclotron frequencies. Section 4 shortly addresses the implications of our study for future radio observations of Tau Bootis b. Section 5 presents our conclusions.

\section{MODEL DESCRIPTION}

\subsection{Upper Atmosphere Modelling}

In Weber et al. (2017), we used upper atmosphere profiles from hydrodynamic models which include heating by stellar XUV radiation, ionization and dissociation of hydrogen. This is a valid approach for most hot Jupiters which have expanded and efficiently escaping atmospheres.

In cases of extremely massive planets, an upper atmosphere in the hydrodynamic regime is hardly possible, and thus it is reasonable to apply equilibrium equations for the atmospheric parameters (see the discussion in Section 4). We derive these equations from the hydrodynamic system in Erkaev et al. (2016) by neglecting terms with time derivatives and bulk velocities. The equations for pressure $P$ and temperature $T$ are as follows

$$
\begin{gathered}
\frac{\partial P}{\partial R}=-\rho \frac{\partial U}{\partial R}, \\
Q_{\mathrm{EUV}}-Q_{\mathrm{cool}}+\frac{\partial}{R^{2} \partial R}\left(R^{2} \chi \frac{\partial T}{\partial R}\right)=0 .
\end{gathered}
$$

Here, $R$ is the distance from the planet, $U$ is the gravitational potential, $\chi$ is the thermal conductivity of the atmosphere (Watson et al. 1981), given by

$\chi=4.45 \cdot 10^{4}\left(\frac{T}{1000 \mathrm{~K}}\right)^{0.7}$,

$Q_{\mathrm{EUV}}$ is the stellar EUV (extreme ultraviolet) volume heating rate

$Q_{\mathrm{EUV}}=\eta \sigma_{\mathrm{EUV}}\left(n_{\mathrm{H}}+n_{\mathrm{H}_{2}}\right) \phi_{\mathrm{EUV}}$,

$\eta$ is the ratio of the net local heating rate to the rate of the stellar radiative absorption in the planetary atmosphere (assumed to be $15 \%$, in agreement with Shematovich et al. 2014) and $Q_{\text {cool }}$ is the cooling rate. The latter consists of the Lyman- $\alpha\left(Q_{\mathrm{L}_{\alpha}}\right)$,

$Q_{\mathrm{L}_{\alpha}}=7.5 \cdot 10^{-19} n_{\mathrm{e}} n_{\mathrm{H}} \exp (-118348 \mathrm{~K} / T)$,

the $\mathrm{H}$ collision ionization $\left(Q_{\mathrm{ci}}\right)$, Bremsstrahlung $\left(Q_{\mathrm{BS}}\right)$ and $\mathrm{H}_{+}$ radiative recombination $\left(Q_{\text {rec }}\right)$ cooling processes, i.e. $Q_{\text {cool }}=$ $Q_{\mathrm{L}_{\alpha}}+Q_{\mathrm{ci}}+Q_{\mathrm{BS}}+Q_{\mathrm{rec}}$. The quantity $\sigma_{\mathrm{EUV}}$ is the cross section of EUV absorption.

The cooling rates $Q_{\mathrm{ci}}, Q_{\mathrm{BS}}$ and $Q_{\text {rec }}$ are taken from Glover $\&$ Jappsen (2007). $\phi_{\mathrm{EUV}}$ is the function describing the EUV flux absorption in the atmosphere

$\phi_{\mathrm{EUV}}=\frac{1}{2} \int_{0}^{\pi / 2+\arccos (1 / r)} J_{\mathrm{EUV}}(r, \theta) \sin (\theta) d \theta$.

Here, $J_{\mathrm{EUV}}(r, \theta)$ is a function of spherical coordinates that describes the spatial variation of the EUV flux due to the atmospheric absorption (Erkaev et al. 2015), $r$ corresponds to the radial distance from the planetary center normalized to planetary photospheric radius $R_{0}$.
The mass density, $\rho$, and the pressure, $P$, are related to the species densities as follows:

$\rho=m_{\mathrm{H}}\left(n_{\mathrm{H}}+n_{\mathrm{H}^{+}}\right)+m_{\mathrm{H}_{2}}\left(n_{\mathrm{H}_{2}}+n_{\mathrm{H}_{2}^{+}}\right)$,

$P=\left(n_{\mathrm{H}}+n_{\mathrm{H}^{+}}+n_{\mathrm{H}_{2}}+n_{\mathrm{H}_{2}^{+}}+n_{\mathrm{e}}\right) k T$,

where $k$ is the Boltzmann constant, and $m_{\mathrm{H}}$ and $m_{\mathrm{H}_{2}}$ are the masses of the hydrogen atoms and molecules, respectively.

Steady-state densities of atomic and molecular hydrogen, ions and electrons satisfy the following algebraic equations

$$
\begin{gathered}
v_{\mathrm{H}} n_{\mathrm{H}}+v_{\mathrm{Hcol}} n_{\mathrm{e}} n_{\mathrm{H}}-\alpha_{\mathrm{H}} n_{\mathrm{e}} n_{\mathrm{H}^{+}}=0, \\
-v_{\mathrm{H}_{2}} n_{\mathrm{H}_{2}}-v_{\mathrm{dis}} n_{\mathrm{H}_{2}} n+\gamma_{\mathrm{H}} n n_{\mathrm{H}}^{2}=0, \\
v_{\mathrm{H}_{2}} n_{\mathrm{H}_{2}}-\alpha_{\mathrm{H}_{2}} n_{\mathrm{e}} n_{\mathrm{H}_{2}^{+}}=0 .
\end{gathered}
$$

The electron density is determined by the quasi-neutrality condition

$$
n_{\mathrm{e}}=n_{\mathrm{H}^{+}}+n_{\mathrm{H}_{2}^{+}}
$$

and the total hydrogen number density is a sum of the partial densities

$$
n=n_{\mathrm{H}}+n_{\mathrm{H}^{+}}+n_{\mathrm{H}_{2}}+n_{\mathrm{H}_{2}^{+}} .
$$

The term $\alpha_{\mathrm{H}}$ is the recombination rate related to the reaction $\mathrm{H}^{+}+e \rightarrow \mathrm{H}$ of $4 \times 10^{-12}(300 \mathrm{~K} / T)^{0.64} \mathrm{~cm}^{3} \mathrm{~s}^{-1}, \alpha_{\mathrm{H}_{2}}$ is the dissociation rate of $\mathrm{H}_{2}^{+}+e \rightarrow \mathrm{H}+\mathrm{H}: \alpha_{\mathrm{H}_{2}}=2.3 \times 10^{-8}(300 \mathrm{~K} / T)^{0.4} \mathrm{~cm}^{3}$ $\mathrm{s}^{-1}, v_{\text {diss }}$ is the thermal dissociation rate of $\mathrm{H}_{2} \rightarrow \mathrm{H}+\mathrm{H}: 1.5 \cdot 10^{-9}$ $\exp (-49000 \mathrm{~K} / T), \gamma_{\mathrm{H}}$ is the rate of the reaction $\mathrm{H}+\mathrm{H} \rightarrow \mathrm{H}_{2}: \gamma_{\mathrm{H}}=$ $8.0 \cdot 10^{-33}(300 \mathrm{~K} / \mathrm{T})^{0.6}$ (Yelle 2004).

The term $v_{\mathrm{H}}$ is the hydrogen ionization rate, and $v_{\mathrm{H}_{2}}$ is the ionization rate of molecular hydrogen (Storey \& Hummer 1995; Murray-Clay et al. 2009),

$$
v_{\mathrm{H}}=5.9 \cdot 10^{-8} \phi_{\mathrm{EUV}}{ }^{-1}, \quad \nu_{\mathrm{H}_{2}}=3.3 \cdot 10^{-8} \phi_{\mathrm{EUV}}{ }^{-1},
$$

and $v_{\mathrm{Hcol}}$ is the collisional ionization rate (Black 1981), $v_{\mathrm{Hcol}}=$ $5.9 \cdot 10^{-11} T^{1 / 2} \exp (-157809 \mathrm{~K} / T)$.

It turns out that the $\mathrm{H}_{2}^{+}$density is much smaller than that of other species. This allows us to neglect $n_{\mathrm{H}_{2}^{+}}$in the equations for the electron and total densities (Equations 12 and 13). With this assumption, we solve the Equations (9-13) and express the total, molecular, ion and electron densities through one quantity - the atomic hydrogen density. Substituting these expressions to (8) and (7), we solve finally two ordinary differential equations $(1,2)$.

Table 1 summarizes the Tau Bootis b system parameters. Table 2 shows the XUV fluxes at orbital distances of 0.046, 0.1 and $0.2 \mathrm{AU}$ from Tau Bootis as well as the temperature $T_{0}$ and pressure $P_{0}$ at the photosphere.

The lower boundary is set at the planetary photospheric radius $R_{0}$. For all cases, and throughout this study, we consider that $R_{0}$ lies at an atmospheric pressure $P_{0}$ of about $100 \mathrm{mbar}$. This is justified by the fact that, for an $\mathrm{H}_{2}$-dominated atmosphere considering a clear atmosphere and taking into account $\mathrm{H}_{2}$ Rayleigh scattering, $\mathrm{H}_{2}-\mathrm{H}_{2}$ collisional-induced absorption, alkali lines, and solar-abundance molecular bands, the optical depth at visible wavelengths is unity at a pressure level of about 100 mbar (Lammer et al. 2016; Fossati et al. 2017; Cubillos 2016; Cubillos et al. 2017). The atmospheric temperature $T_{0}$ at $R_{0}$ is assumed to be the equilibrium temperature at the planet's orbital location (see Table 2).

To infer the XUV flux of Tau Bootis, we have first derived 
Table 1. Tau Bootis system parameters. The stellar and planetary parameters are from http://exoplanet.eu/catalog/tau_boo_b/, accessed on 01.06.2018.

\begin{tabular}{ll}
\hline Planet & \\
\hline Orbital distance & $0.046 \mathrm{AU}$ \\
Distance from Earth & $15.6 \mathrm{pc}$ \\
Mass & $5.84 M_{\mathrm{J}}$ \\
Radius $R_{0}$ & $1.06 R_{\mathrm{J}}$ \\
Dipole moment $\mathcal{M}$ & $0.76 \mathcal{M}_{\mathrm{J}}{ }^{*}$ \\
\hline \hline Star & Tau Bootis \\
\hline Spectral type & F7 V \\
Mass & $1.3 M_{\text {Sun }}$ \\
Radius & $1.331 R_{\text {Sun }}$ \\
Age & $2.52 \mathrm{Gyr}$ \\
\hline \hline *(Grießmeier et al. 2007a) & \\
\hline
\end{tabular}

the chromospheric emission at the core of the CaII H\&K lines, for which high-quality observations are available, and converted it to an XUV flux value. We have first computed the synthetic spectral energy distribution of the stellar photosphere employing the LLmodels stellar atmosphere code (Shulyak et al. 2004) to which we have then added various levels of emission at the core of the CaII H\&K lines, as described in Fossati et al. (2015), until we could fit the optical observations obtained with the ESPADONS spectropolarimeter available in the CFHT archive. The star Tau Bootis is located much closer than 100 pc, thus interstellar medium absorption does not significantly affect the CaII line core emission (Fossati et al. 2017). In this way, we obtained an integrated CaII chromospheric emission flux at a distance of $1 \mathrm{AU}$ from the star of $0.05 \mathrm{~W} / \mathrm{m}^{2}$. We have converted this flux into a Ly $\alpha$ flux and then into an XUV flux using the scaling relations of Linsky et al. (2013, 2014), obtaining an XUV flux of $17.5 \mathrm{~W} / \mathrm{m}^{2}$ at the distance of the planet. Being based on two scaling relations, this value has an uncertainty of the order of $50 \%$ (e.g. Fossati et al. 2015).

The temperature peak for Tau Bootis $b$ which would be needed for hydrodynamic escape is about $\sim 100000 \mathbf{K}$, i.e. one order of magnitude higher than for the previously studied hot Jupiter HD 209458b. Such a high temperature is not realistic, because cooling processes can reduce it immediately. In case of a supermassive planet, the temperature peak is so high that the cooling processes make a significant contribution. Therefore the cooling processes reduce the temperature peak below the critical level needed for a hydrodynamic escape regime. When trying to run a hydrodynamic code with cooling for the case of a supermassive planet, there is no radial acceleration of the atmospheric particles because the temperature is not sufficiently high for such acceleration against the gravitational forces. We plan to investigate this effect in detail within a follow-up study.

Figure 1 shows the number density profiles of $\mathrm{H}, \mathrm{H}_{2}, \mathrm{H}^{+}$and $\mathrm{H}_{2}^{+}$(i.e., neutrals and plasma) at 0.046, 0.1 and $0.2 \mathrm{AU}$ for Tau Bootis $\mathrm{b}$ (upper panels and lower left panel) and the temperature profile for the different orbital distances (right lower panel). The atmosphere is not expanding hydrodynamically and very favourable conditions for the CMI can be expected. The strong gravity and the radiative cooling keep the atmosphere compact and we expect similar conditions as for e.g. Jupiter, with large regions of depleted plasma between the exobase and the magnetopause.

By estimating the hydrogen scale height $\left(H=k T_{\mathrm{exo}} / m_{\mathrm{H}} g\right.$,
Table 2. Stellar XUV radiation at different orbital separations for Tau Bootis. Planetary atmospheric temperature and pressure $R_{0}$ of a Tau Bootis b-like planet at different orbital distances.

\begin{tabular}{|c|c|c|c|}
\hline $\begin{array}{l}\text { Orbital distance } \\
{[\mathrm{AU}]}\end{array}$ & $\begin{array}{l}\mathrm{XUV} \\
{\left[\mathrm{W} / \mathrm{m}^{2}\right]}\end{array}$ & $\begin{array}{l}\text { Temperature } \\
T_{0}[\mathrm{~K}]\end{array}$ & $\begin{array}{l}\text { Pressure } \\
P_{0} \text { [mbar] }\end{array}$ \\
\hline 0.046 & 17.53 & 1700 & 100 \\
\hline 0.1 & 3.71 & 1150 & 100 \\
\hline 0.2 & 0.93 & 810 & 100 \\
\hline
\end{tabular}

Table 3. Stellar wind parameters (velocity $v_{\mathrm{sw}}$ and density $n_{\mathrm{sw}}$ ) of Tau Bootis at different orbital distances.

\begin{tabular}{|c|c|c|}
\hline $\begin{array}{l}\text { Orbital distance } \\
{[\mathrm{AU}]}\end{array}$ & $v_{\mathrm{sw}}[\mathrm{km} / \mathrm{s}]$ & $n_{\mathrm{sw}}\left[\mathrm{m}^{-3}\right]$ \\
\hline 0.046 & 272 & $4.16 \cdot 10^{10}$ \\
\hline 0.1 & 340 & $6.04 \cdot 10^{9}$ \\
\hline 0.2 & 408 & $1.22 \cdot 10^{9}$ \\
\hline
\end{tabular}

where $k$ is the Boltzmann constant, $T_{\text {exo }}$ the temperature at the exobase, $m_{\mathrm{H}}$ the hydrogen mass and $g$ the gravitational acceleration) at the exobase level of Tau Boo b and Jupiter we obtain $365.43 \mathrm{~km}$ and $4443.2 \mathrm{~km}$, respectively. The distance between exobase and magnetopause for Jupiter is $40.9 R_{\mathrm{J}}$ or 7.8 $\cdot 10^{3}$ in scale heights and 3.6 $R_{\mathrm{p}}$ or 59.7 in scale heights for Tau Bootis b. One can expect that the exosphere density of Tau Boo b decreases fast so that the plasmasphere between the exobase and possible magnetopause distances will be populated mainly with stellar wind plasma. Of course the stellar wind plasma at Tau Bootis b orbit is much higher than at 5.2 AU but this poses no problem for the propagation of possibly generated radio waves (Grießmeier et al. 2007b). Therefore, the upper atmospheremagnetosphere configuration and the related plasmasphere are more comparable with Jupiter in the Solar System, making the production of radio waves by the CMI and propagation in that environment more likely compared to a classical hot Jupiters like HD 209458b or HD $189733 b$.

\subsection{Comparison between exobase and magnetopause standoff} distance

The magnetopause standoff distances are calculated from (e.g. Grießmeier et al. 2004; Khodachenko et al. 2012; Kislyakova et al. 2014)

$R_{\mathrm{S}}=\left(\frac{\mathcal{M}^{2} \mu_{0} f_{0}^{2}}{8 \pi^{2} \rho_{\mathrm{sw}} v_{\mathrm{sW}}^{2}}\right)^{\frac{1}{6}}$.

Here, $\mu_{0}$ is the vacuum permeability and $f_{0}=1.22$ is a form factor for the magnetopause shape including the influence of a magnetodisk (Khodachenko et al. 2012). $v_{\mathrm{sw}}$ is the stellar wind velocity corrected for the orbital motion of the planet. $\rho_{\mathrm{sw}}$ is the stellar wind density and $\mathcal{M}$ is the magnetic moment. Table 3 summarizes the stellar wind parameters from the model of Grießmeier et al. (2007a,b) for the different orbital distances. Figure 2 shows the exobase levels $R_{\mathrm{e}}$ compared to the magnetopause standoff distances $R_{\mathrm{S}}$ as a function of orbital distance for the magnetic moments as predicted by Grießmeier et al. (2007a) for Tau Bootis b and the 

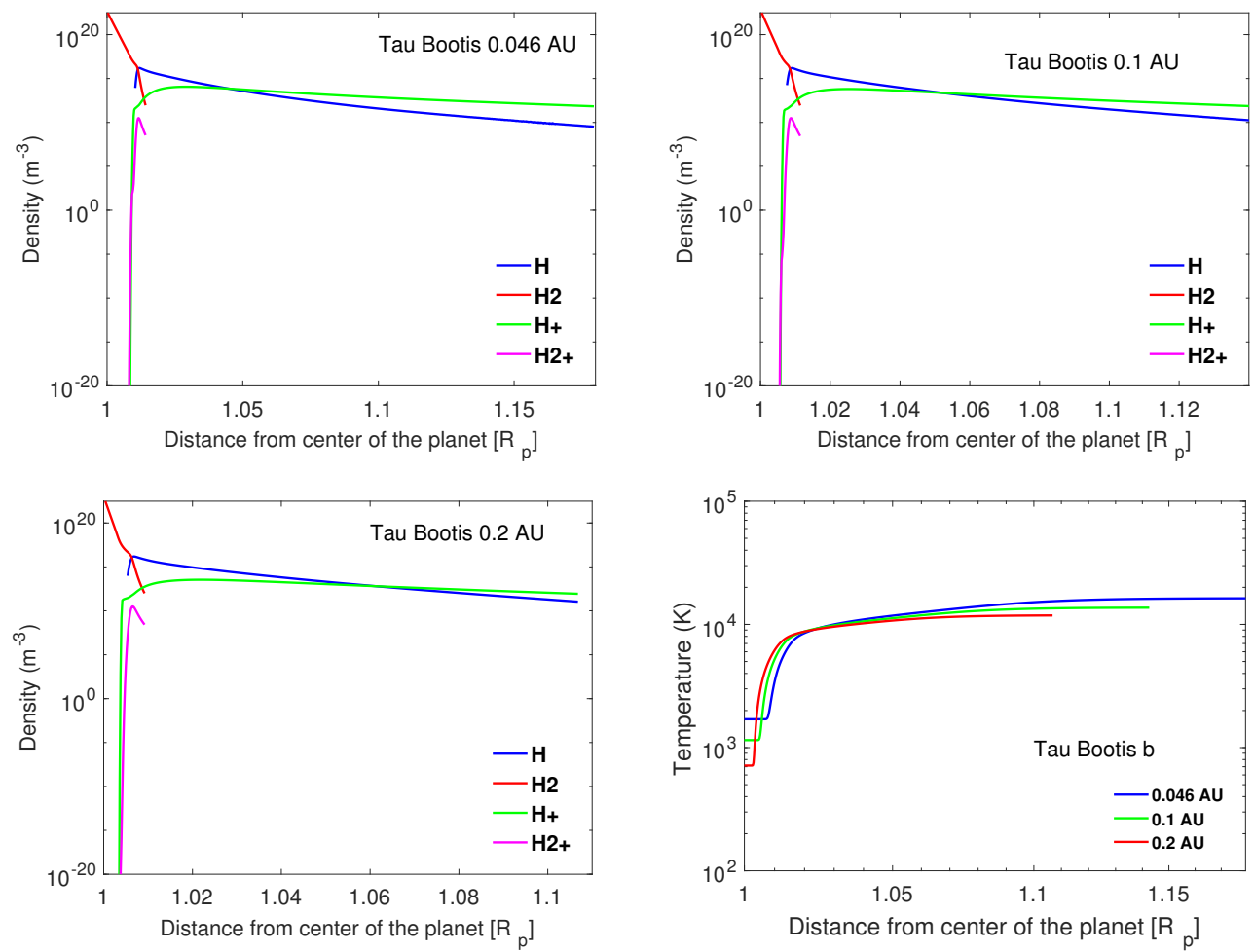

Figure 1. Top panels and bottom-left panel: $\mathrm{H}, \mathrm{H}_{2}, \mathrm{H}^{+}$and $\mathrm{H}_{2}^{+}$densities at orbital distances of $0.046,0.1$ and 0.2 AU for Tau Bootis b around the F7V star Tau Bootis. Note that we only plot the regions up to the exobase level. Bottom-right panel: temperature profiles for $0.046,0.1$ and 0.2 AU.

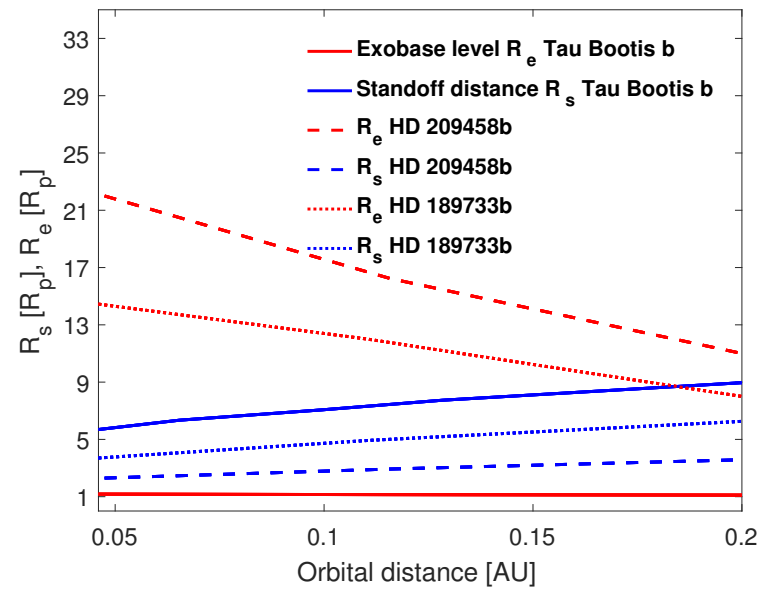

Figure 2. Exobase levels $R_{\mathrm{e}}$ compared to the magnetopause standoff distances $R_{\mathrm{S}}$ as a function of orbital distance for the magnetic moments as predicted by Grießmeier et al. (2007a) for Tau Bootis b and the planets studied in Weber et al. (2017).

planets studied in Weber et al. (2017), i.e. HD $209458 b$ and HD 189733b. For Tau Bootis $b$, the exobase is very close to the planet (at 1.18, 1.14 and 1.11 $R_{\mathrm{p}}$ for $0.046,0.1$ and $0.2 \mathrm{AU}$, respectively) and the magnetospheric cavity for the CMI to work can be expected to be very large. For all orbital distances between 0.046 and $0.2 \mathrm{AU}$ the exobase is smaller than the standoff distance. For HD $209458 b$ and HD 189733b, assuming the same XUV flux as for Tau Bootis b, for each of the investigated orbits the exobase is larger than the magnetopause standoff distance.

\section{PLASMA ENVIRONMENT AND CORRESPONDING FREQUENCIES}

For the cyclotron frequency, we test three different hypotheses for the planetary magnetic moment: (a) we use the magnetic field strengths predicted by Grießmeier et al. (2007a), (b) we compare to the value of Reiners \& Christensen (2010), and (c) we also compare to results using the rotation-independent value of Grießmeier et al. (2011). To calculate the corresponding cyclotron frequency $f_{\mathrm{c}}$ we use

$f_{\mathrm{c}}=\frac{1}{2 \pi} \frac{e \cdot B}{m_{\mathrm{e}}}$.

Here, $e$ is the electron charge, $B$ the magnetic field strength and $m_{\mathrm{e}}$ the electron mass. The relation between magnetic dipole moment and the maximum magnetic field strength at the pole is given by

$B=\frac{\mu_{0}}{4 \pi} \frac{\mathcal{M}}{R_{\mathrm{p}}^{3}}$,

where $R_{\mathrm{p}}$ is the planetary radius, $\mu_{0}$ is the vacuum permeability and $\mathcal{M}$ is the magnetic moment. The plasma frequency is calculated via

$f_{\mathrm{p}}=\frac{1}{2 \pi} \sqrt{\frac{e^{2} n_{\mathrm{e}}}{m_{\mathrm{e}} \varepsilon_{0}}}$,

with $n_{\mathrm{e}}$ the electron density and $\varepsilon_{0}$ the vacuum permittivity. For the electron density at Tau Bootis b, results from Section 2.1 are evaluated. 


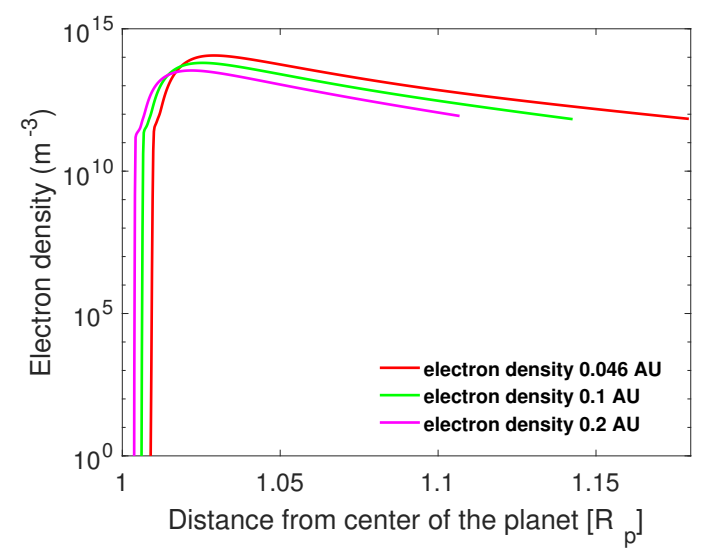

Figure 3. Electron density profiles of a Tau Bootis b-like planet at orbital distances of 0.046 (red), 0.1 (green) and $0.2 \mathrm{AU}$ (magenta). The curves end at the exobase level.

The plasma and cyclotron frequency are calculated to check whether the condition for the Cyclotron Maser Instability to work (i.e. $f_{\mathrm{p}} \ll f_{\mathrm{c}}$ ) is fulfilled (Weber et al. 2018, 2017).

Grießmeier et al. (2007a) predicted the magnetic moment of Tau Bootis b to be $0.76 \mathcal{M}_{\mathrm{J}}$, where $\mathcal{M}_{\mathrm{J}}$ is the Jovian magnetic moment. For the magnetic moment, we also considered the model by Reiners \& Christensen (2010), in which tidal locking has no influence. They predict a dipole magnetic field strength at the pole of $58 \mathrm{G}$. This corresponds to a magnetic moment of $1.25 \cdot 10^{28} \mathrm{Am}^{2} \approx$ $8 \mathcal{M}_{\mathrm{J}}$. The value found in Grießmeier et al. (2011) corresponds to $\approx$ $20.3 \mathrm{G}$ and a magnetic moment of $2.8 \mathcal{M}_{\mathrm{J}}$.

A recent study by Yadav \& Thorngren (2017) states that magnetic moments of up to 10 times stronger than those of Jupiter can be expected for hot Jupiters regardless of their age, provided that the energy of the stellar radiation is deposited in the planetary center. With the processes suggested here this is, however, not a realistic assumption. In the more realistic case of energy deposition in the planet's outer layers, the extra energy would reduce the thermal gradient within the planet or even invert it. This would reduce the convection in the planet. Rather than strengthening the planetary dynamo, this would weaken it, and could even shut it down altogether.

Figure 3 shows the electron density profiles of Tau Bootis $b$ placed at $0.046 \mathrm{AU}$ (red line), $0.1 \mathrm{AU}$ (green line) and $0.2 \mathrm{AU}$ (magenta line). Figure 4 shows the cyclotron frequency (green, blue and magenta dashed lines) and the plasma frequency at different orbital distances (red, green and magenta lines). The cyclotron frequency was calculated from equation (16) assuming a dipolar magnetic field, using the predicted magnetic moments of $0.76 \mathcal{M}_{\mathrm{J}}$ from Grießmeier et al. (2007a) (dashed green line, G2007), the predicted $58 \mathrm{G}\left(7.5 \mathcal{M}_{\mathrm{J}}\right.$ ) from Reiners \& Christensen (2010) (dashed blue line, denoted by RC2010) and the value of $2.8 \mathcal{M}_{\mathrm{J}}$ found in Grießmeier et al. (2011) (dashed magenta line). Radio emission is generated at frequencies close to the local cyclotron frequency of the electrons. Only if the cyclotron frequency exceeds the local plasma frequency $\left(f_{\mathrm{p}} / f_{\mathrm{c}} \leqslant 0.4\right.$; (Grießmeier et al. 2007a)), the condition for the generation of radio waves is fulfilled and radio waves with a frequency $f_{\mathrm{c}}>f_{\mathrm{p}}$ can be generated. Radio waves generated at a hypothetical distance $R_{1}$ can escape from their generation region through the planetary atmosphere/ionosphere if and only if the cyclotron frequency at the distance $R_{1}$ is higher than the plasma frequency for all distances $R_{1}<R<\infty$.

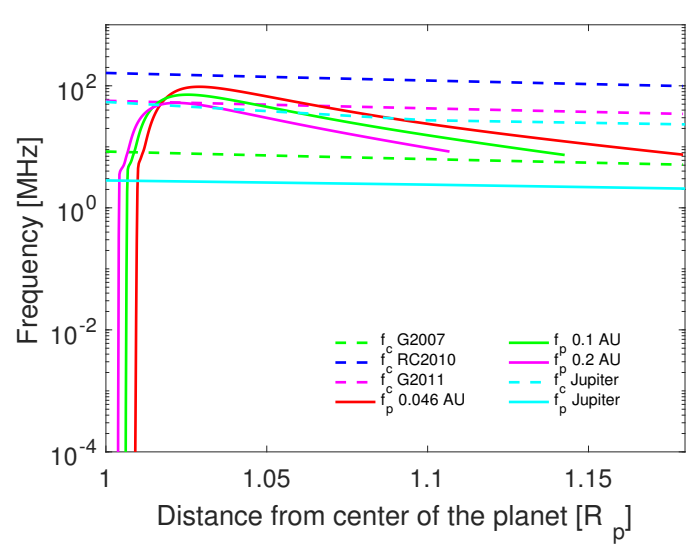

Figure 4. Comparison of cyclotron frequency to plasma frequency. The red, green and magenta lines correspond to the plasma frequency at 0.046, 0.1 and $0.2 \mathrm{AU}$, respectively, and stop at the exobase levels. The dashed green line shows the cyclotron frequency corresponding to a magnetic moment as predicted by Grießmeier et al. (2007a). The dashed blue line shows the cyclotron frequency corresponding to a polar magnetic field strength of 58 G, as predicted by Reiners \& Christensen (2010). The dashed magenta line corresponds to the prediction by Grießmeier et al. (2011). The solid and dashed cyan lines correspond to the plasma and cyclotron frequencies at Jupiter, respectively. The plotting range stops at the largest exobase level $\left(1.18 R_{\mathrm{p}}\right.$ at $\left.0.046 \mathrm{AU}\right)$.

All curves in Figure 4 stop at the exobase level. The range of the plot stops at the exobase level for $0.046 \mathrm{AU}$, i.e. $1.18 R_{\mathrm{p}}$. In the regions up to the exobase level for the magnetic field cases RC2010 and G2011 possibly generated radio waves can escape but not for G2007. However, the exobase levels are very low, i.e. $1.18 R_{\mathrm{p}}, 1.14 R_{\mathrm{p}}$ and $1.11 R_{\mathrm{p}}$ for $0.046,0.1$ and $0.2 \mathrm{AU}$, respectively. This means that the atmosphere is very compact. Beyond the exobase the conditions for the CMI are very likely much better. Thus, each magnetic field case should yield the possibility of generation and escape for radio emission. Only for the case $\mathbf{G 2 0 0 7}$ emission generated in the small region below the exobase levels would not be able to escape. Beyond the exobase, all cyclotron frequencies should exceed the plasma frequencies.

Figure 5 shows the ratios of plasma to cyclotron frequency for the orbits $0.046,0.1$ and $0.2 \mathrm{AU}$, respectively. For each orbit the corresponding plasma frequency is compared with the different cyclotron frequencies corresponding to the magnetic field cases as discussed above, i.e. the case G2007, corresponding to the magnetic moment as predicted by Grießmeier et al. (2007a), the case RC2010, corresponding to the magnetic moment as predicted by Reiners \& Christensen (2010) and the case G2011, corresponding to the magnetic moment as predicted by Grießmeier et al. (2011). The solid black line indicates the maximum frequency ratio of 0.4 for the CMI to work (see Grießmeier et al. 2007b; Weber et al. 2017). The same argument as above holds, i.e. because the plotting range stops at the exobase levels, the conditions for the CMI in these small region are not good only for the case G2007. Beyond the exobase, for every magnetic field case, the plasma frequency should be below the cyclotron frequency almost everywhere within the magnetosphere. This can be seen very well by the decreasing trend of the frequency ratios for all cases. When there is no hydrodynamic atmospheric outflow then exobase densities are always similar. Thus, we can expect a situation comparable to Jupiter in the solar system at 5.2 AU, where the frequency ratios are well below the indicated maximum of 0.4 because the plasma 

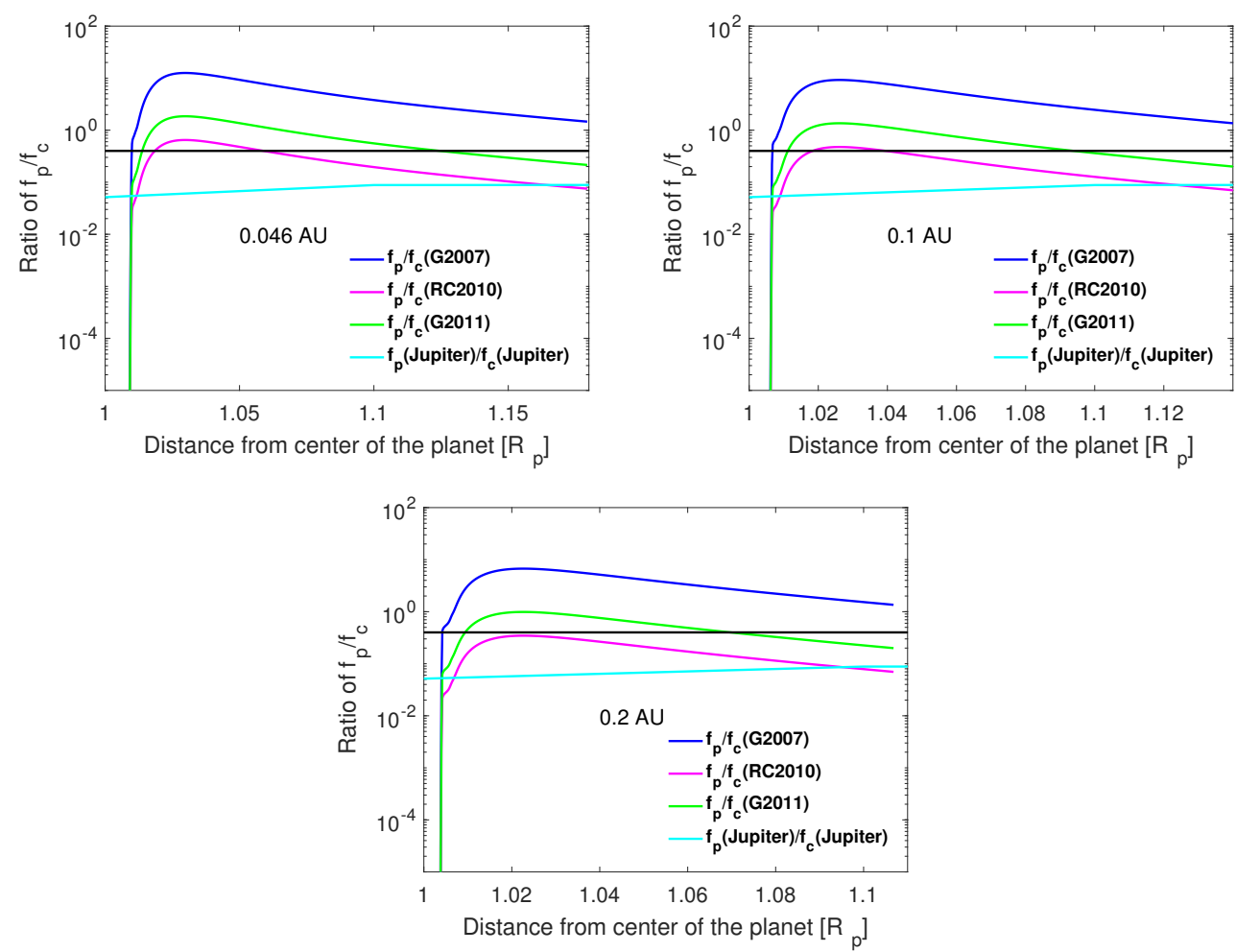

Figure 5. Ratio of plasma to cyclotron frequency for Tau Bootis b for different orbital distances. Upper-left panel: 0.046 AU. Upper-right panel: 0.1 AU. Lower panel: $0.2 \mathrm{AU}$. The black solid line indicates the maximum ratio of 0.4 for the CMI to work. Note that the curves end at the exobase levels.

density decreases very fast beyond the exobase level. For details on the Jovian plasma environment we refer to e.g. Prasad \& Capone (1971); Machida \& Nishida (1978); Luhmann \& Walker (1980); Yelle et al. (1996); Stallard et al. (2001).

Table 4 gives a summary of the possibility for generation and escape of potential radio emission for the cases studied in this paper. The + signs indicate that generation or escape is possible. The case of Jupiter $\left(1 \mathcal{M}_{\mathrm{J}}\right)$ is intended as comparison to the cases studied here. To compare the results of the current study with our former findings, Table 5 shows the same summary as for Tau Bootis b, but for the planets HD 189733 b and HD 209458b from Weber et al. (2017). In the version of the table used here we filled the gaps in the table of Weber et al. (2017).

\section{IMPLICATIONS FOR FUTURE OBSERVATION CAMPAIGNS TARGETING TAU BOOTIS B}

Radio emission from the Tau Bootis b system hasn't been detected yet, even though we find, in agreement with several former studies (e.g. Farrell et al. 2003; Lazio \& Farrell 2007; Grießmeier et al. 2005, 2006b; Grießmeier 2007; Grießmeier et al. 2007b,a, 2011), that it should be a good candidate for successful observations. Radio fluxes were predicted to be in the sensitivity range of several radio telescopes, e.g. UTR-2 and LOFAR. Grießmeier et al. (2011) compared the rotation independent model of Reiners \& Christensen (2010) to their model where the planetary magnetic moment is dependent on rotation, and thus tidal locking has an influence. They find radio fluxes of 180 and $300 \mathrm{mJy}$ for the rotation independent and the rotation dependent model, respectively. Since the cyclotron frequencies calculated in this paper are based on the magnetic moment predictions from these models, the maximum emission frequency and the radio flux densities are the same. Thus, we conclude that radio emission from Tau Bootis b should be detectable with LOFAR, UTR-2, the upcoming SKA (Square Kilometer Array), NenuFAR (New Extension in Nançay Upgrading LOFAR, currently under construction) (Grießmeier et al. 2011) and GURT (under construction in Kharkov, Ukraine Konovalenko et al. 2016). Also the VLA (Very Large Array), the LWA (Long Wavelength Array) and the GMRT (Giant Metrewave Radio Telescope) should be sensitive enough to allow a detection (Grießmeier et al. 2011).

Finally, we note that for HD $209458 \mathrm{~b}$ radiative cooling can be neglected, whereas for HD 189733b it already has an effect. For details we refer to the study by Salz et al. (2015), who described HD $189733 \mathrm{~b}$ as an intermediate case. We plan to perform a similar study as for Tau Bootis b for HD $189733 \mathrm{~b}$. Recently, Lalitha et al. (2018) studied the atmospheric mass loss of four close-in planets around the very active stars Kepler63, Kepler-210, WASP-19, and HAT-P-11. They found the XUV luminosities of these stars to be orders of magnitude higher than for the Sun. Lalitha et al. (2018) compared the four studied planets with HD 209458b and HD 189733b. All planets suffer extreme atmospheric mass loss due to the strong XUV radiation from their host stars, very likely leading to the extended upper atmospheres and ionospheres, which might inhibit the escape of possibly generated radio emission. 
Table 4. Summary of possibility for generation and escape of radio emission for the case studied in this paper. The case of Jupiter with $1 \mathcal{M}_{\mathrm{J}}$ is shown for comparison. The numbers in brackets are the maximum emission frequencies in $\mathrm{MHz}$.

\begin{tabular}{|c|c|c|c|}
\hline Magnetic moment $\left[\mathcal{M}_{\mathrm{J}}\right]$ & $0.046 \mathrm{AU}$ & $0.1 \mathrm{AU}$ & $0.2 \mathrm{AU}$ \\
\hline & Tau Bootis & & \\
\hline \multirow{2}{*}{$\begin{array}{l}0.76 \mathcal{M}_{\mathrm{J}} \approx \approx 10.5 \mathrm{MHz}, \\
\mathrm{G} 2007)\end{array}$} & generation: + & generation: + & generation: + \\
\hline & escape: + & escape: + & escape: + \\
\hline $1 \mathcal{M}_{\mathrm{J}}(\approx 23.9 \mathrm{MHz})$ & $\begin{array}{l}\text { generation: + } \\
\text { escape: }+\end{array}$ & $\begin{array}{l}\text { generation: + } \\
\text { escape: }+\end{array}$ & $\begin{array}{l}\text { generation: + } \\
\text { escape: }+\end{array}$ \\
\hline \multirow{2}{*}{$\begin{array}{l}2.8 \mathcal{M}_{\mathrm{J}} \\
\mathrm{G} 2011)\end{array} \quad \approx 11 \mathrm{MHz}$} & generation: + & generation: + & generation: + \\
\hline & escape: + & escape: + & escape: + \\
\hline \multirow{2}{*}{$\begin{array}{l}7.5 \mathcal{M}_{\mathrm{J}}(\approx 161 \mathrm{MHz}, \\
\mathrm{RC} 2010)\end{array}$} & generation: + & generation: + & generation: + \\
\hline & escape: + & escape: + & escape: + \\
\hline
\end{tabular}

Table 5. Summary of possibility for generation and/or escape of radio waves for the planets HD 209458b and HD 189733b (Weber et al. 2017). The numbers in brackets are the maximum emission frequencies in MHz.

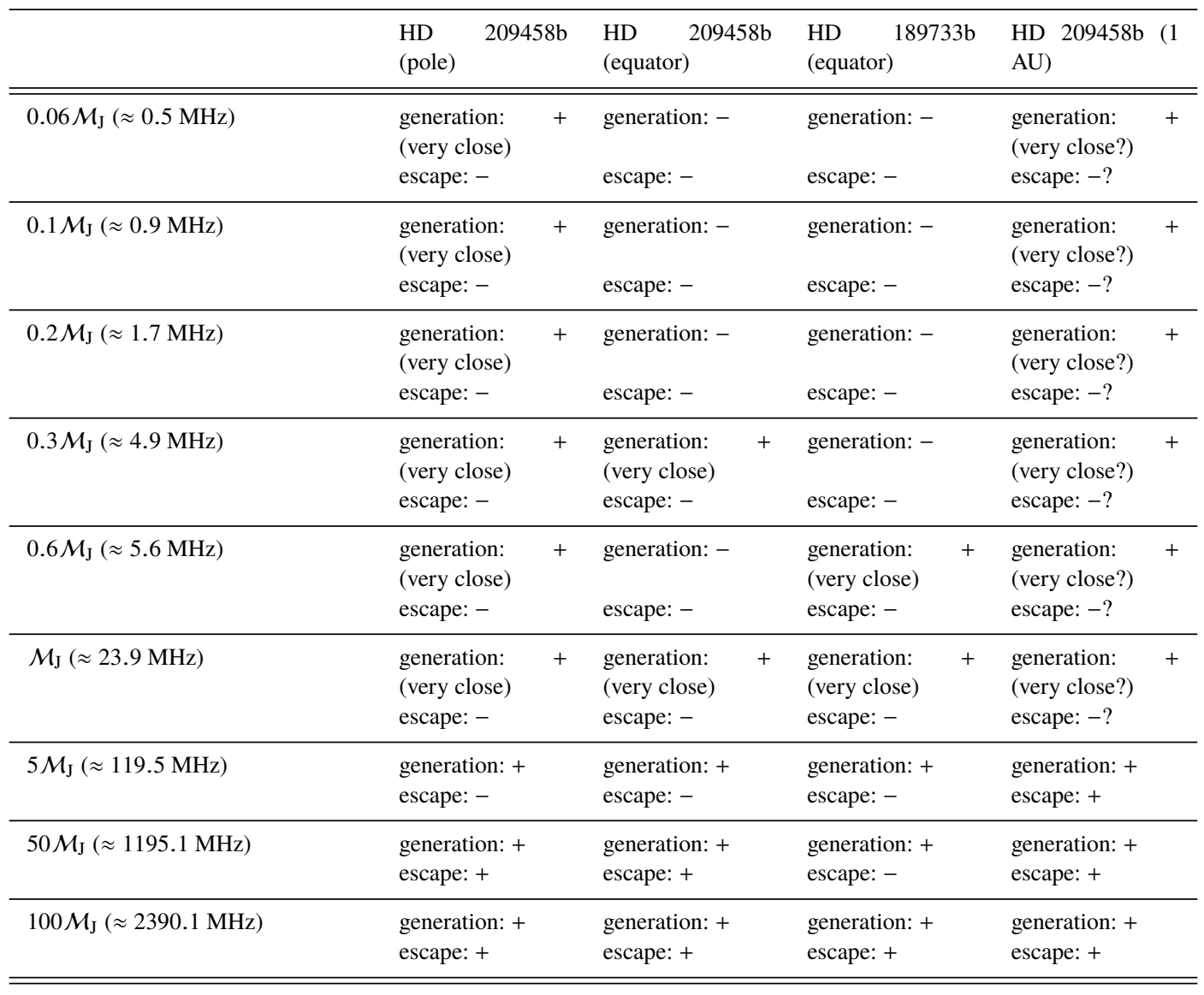

\section{CONCLUSIONS}

We find that the supermassive Hot Jupiter Tau Bootis b is clearly more favourable for the CMI than hot Jupiters like HD 209458b or HD 189733b studied previously. The main issue with the latter planets is the extended upper atmosphere and ionosphere, where exobase distances exceed the magnetospheric standoff distance, and upper atmospheres are in a hydrodynamic state for orbits $<0.2 \mathrm{AU}$ for Sun-like stars and $<0.5 \mathrm{AU}$ for more active stars (Weber et al. 2017).

In general, we find that supermassive hot Jupiters like Tau Bootis $b$ are much better candidates for future radio observations than classical hot Jupiters. There is no hydrodynamic outflow and the conditions for the CMI are very good, especially if compared to less massive hot Jupiters like HD 209458b or HD 189733b (0.69 and 
1.142 Jupiter masses, respectively, from http://exoplanet.eu, accessed 01.06.2018), where the atmospheric outflow is hydrodynamic. It is also worth to note that recently Daley-Yates \& Stevens $(2017,2018)$ found the same result for "normal" hot Jupiters as in Weber et al. $(2018,2017)$.

Considering our example planet Tau Bootis b, we can definitely say that objects with $\mathbf{5 . 8 4} M_{\mathrm{J}}$ (Jupiter masses) keep their atmosphere compact due to their large gravity and thus lead to favourable conditions for the CMI. In follow-up studies we will investigate at which mass the transition to these conditions starts. This will include variations of the type of the star or the planetary radius. A further step in our follow-up studies will include an extension of the investigated magnetospheric regions beyond the exobase level up to the magnetopause.

\section{ACKNOWLEDGEMENTS}

C. Weber, H. Lammer and P. Odert acknowledge support from the FWF project P27256-N27 'Characterizing Stellar and Exoplanetary Environments via Modeling of Lyman- $\alpha$ Transit Observations of hot Jupiters'. The authors acknowledge also the support by the FWF NFN projects S11606-N16 'Magnetospheres Magnetospheric Electrodynamics of Exoplanets' and S11607-N16 'Particle/Radiative Interactions with Upper Atmospheres of Planetary Bodies Under Extreme Stellar Conditions'. N. Erkaev and V. Ivanov acknowledge support by the Russian Science Foundation grant No 18-12-00080.

\section{REFERENCES}

Bastian T. S., Dulk G. A., Leblanc Y., 2000, ApJ, 545, 1058

Berger E., et al., 2010, ApJ, 709, 332

Black J. H., 1981, MNRAS, 197, 553

Cubillos P. E., 2016, PhD thesis, University of Central Florida (arXiv: 1604.01320)

Cubillos P., et al., 2017, MNRAS, 466, 1868

Daley-Yates S., Stevens I. R., 2017, Astronomische Nachrichten, 338, 881

Daley-Yates S., Stevens I. R., 2018, preprint, (arXiv: 1806.08147)

Donati J.-F., et al., 2008, MNRAS, 385, 1179

Ergun R. E., Carlson C. W., McFadden J. P., Delory G. T., Strangeway R. J., Pritchett P. L., 2000, ApJ, 538, 456

Erkaev N. V., Lammer H., Odert P., Kulikov Y. N., Kislyakova K. G., 2015, MNRAS, 448, 1916

Erkaev N. V., Lammer H., Odert P., Kislyakova K. G., Johnstone C. P., Güdel M., Khodachenko M. L., 2016, MNRAS, 460, 1300

Fares R., et al., 2010, MNRAS, 406, 409

Farrell W. M., Desch M. D., Zarka P., 1999, J. Geophys. Res., 104, 14025

Farrell W. M., Desch M. D., Lazio T. J., Bastian T., Zarka P., 2003, in Deming D., Seager S., eds, Astronomical Society of the Pacific Conference Series Vol. 294, Scientific Frontiers in Research on Extrasolar Planets. pp 151156

Fossati L., France K., Koskinen T., Juvan I. G., Haswell C. A., Lendl M., 2015, ApJ, 815, 118

Fossati L., et al., 2017, A\&A, 598, A90

Fossati L., Koskinen T., France K., Cubillos P. E., Haswell C. A., Lanza A. F., Pillitteri I., 2018, AJ, 155, 113

Glover S. C. O., Jappsen A.-K., 2007, ApJ, 666, 1

Grießmeier J.-M., 2007, Planet. Space Sci., 55, 530

Grießmeier J.-M., 2018, in Fischer G. e. a., ed., Planetary Radio Emission VIII. Austrian Academy of Sciences Press, Vienna, in press. pp 285-299

Grießmeier J.-M., et al., 2004, A\&A, 425, 753

Grießmeier J.-M., Motschmann U., Mann G., Rucker H. O., 2005, A\&A, 437,717
Grießmeier J., Motschmann U., Glassmeier K., Mann G., Rucker H., 2006a, in Tenth Anniversary of 51 Peg-b: Status of and prospects for hot Jupiter studies. pp 259-266

Grießmeier J.-M., Motschmann U., Khodachenko M., Rucker H. O., 2006b, in Rucker H. O., Kurth W., Mann G., eds, Planetary Radio Emissions VI. p. 571

Grießmeier J.-M., Preusse S., Khodachenko M., Motschmann U., Mann G., Rucker H. O., 2007a, Planet. Space Sci., 55, 618

Grießmeier J.-M., Zarka P., Spreeuw H., 2007b, A\&A, 475, 359

Grießmeier J.-M., Zarka P., Girard J. N., 2011, Radio Science, 46, 0

Hallinan G., Antonova A., Doyle J., Bourke S., Lane C., Golden A., 2008, The Astrophysical Journal, 684, 644

Hallinan G., Sirothia S. K., Antonova A., Ishwara-Chandra C. H., Bourke S., Doyle J. G., Hartman J., Golden A., 2013, ApJ, 762, 34

Hallinan G., et al., 2015, Nature, 523, 568

Herrero E., Morales J. C., Ribas I., Naves R., 2011, A\&A, 526, L10

Khodachenko M. L., et al., 2012, ApJ, 744, 70

Kislyakova K. G., Holmström M., Lammer H., Odert P., Khodachenko M. L., 2014, Science, 346, 981

Konovalenko A., et al., 2016, Experimental Astronomy, 42, 11

Lalitha S., Schmitt J. H. M. M., Dash S., 2018, MNRAS, 477, 808

Lammer H., et al., 2016, MNRAS, 461, L62

Lazio T. J. W., Farrell W. M., 2007, ApJ, 668, 1182

Lazio W. T. J., Farrell W. M., Dietrick J., Greenlees E., Hogan E., Jones C., Hennig L. A., 2004, ApJ, 612, 511

Linsky J. L., France K., Ayres T., 2013, ApJ, 766, 69

Linsky J. L., Fontenla J., France K., 2014, ApJ, 780, 61

Llama J., Jardine M. M., Wood K., Hallinan G., Morin J., 2018, ApJ, 854, 7

Luhmann J. G., Walker R. J., 1980, Icarus, 44, 361

Lynch C. R., Murphy T., Lenc E., Kaplan D. L., 2018, MNRAS,

Machida S., Nishida A., 1978, Planet. Space Sci., 26, 745

Majid W., Winterhalter D., Chandra I., Kuiper T., Lazio J., Naudet C., Zarka P., 2006, in Rucker H. O., Kurth W., Mann G., eds, Planetary Radio Emissions VI. p. 589

Murray-Clay R. A., Chiang E. I., Murray N., 2009, ApJ, 693, 23

Prasad S. S., Capone L. A., 1971, Icarus, 15, 45

Reiners A., Christensen U. R., 2010, A\&A, 522, A13

Route M., Wolszczan A., 2016a, ApJ, 821, L21

Route M., Wolszczan A., 2016b, ApJ, 830, 85

Ryabov V. B., Zarka P., Ryabov B. P., 2004, Planet. Space Sci., 52, 1479

Salz M., Czesla S., Schneider P., Schmitt J., 2015, arXiv preprint arXiv: 1511.09341

Shematovich V. I., Ionov D. E., Lammer H., 2014, A\&A, 571, A94

Shiratori Y., Yokoo H., Saso T., Kameya O., Iwadate K., Asari K., 2006, in Arnold L., Bouchy F., Moutou C., eds, Tenth Anniversary of 51 Peg-b: Status of and prospects for hot Jupiter studies. pp 290-292

Shulyak D., Tsymbal V., Ryabchikova T., Stütz C., Weiss W. W., 2004, A\&A, 428, 993

Stallard T., Miller S., Millward G., Joseph R. D., 2001, Icarus, 154, 475

Storey P. J., Hummer D. G., 1995, MNRAS, 272, 41

Stroe A., Snellen I. A. G., Röttgering H. J. A., 2012, A\&A, 546, A116

Treumann R. A., 2006, A\&ARv, 13, 229

Turner J., Grießmeier J.-M., Zarka P., Vasylieva I., 2017, in Fischer G. e. a., ed., Planetary Radio Emission VIII. Austrian Academy of Sciences Press, Vienna, in press. pp 301-313

Watson A. J., Donahue T. M., Walker J. C. G., 1981, Icarus, 48, 150

Weber C., et al., 2017, MNRAS, 469, 3505

Weber C., et al., 2018, in Fischer G. e. a., ed., Planetary Radio Emission VIII. Austrian Academy of Sciences Press, Vienna, in press. pp 317-329

Williams P. K. G., Berger E., Zauderer B. A., 2013, ApJ, 767, L30

Williams P. K. G., Gizis J. E., Berger E., 2017, ApJ, 834, 117

Winterhalter D., et al., 2006, in Rucker H. O., Kurth W., Mann G., eds, Planetary Radio Emissions VI. p. 595

Yadav R. K., Thorngren D. P., 2017, ApJ, 849, L12

Yelle R. V., 2004, Icarus, 170, 167

Yelle R. V., Young L. A., Vervack R. J., Young R., Pfister L., Sandel B. R., 1996, J. Geophys. Res., 101, 2149

Zarka P., 1998, J. Geophys. Res., 103, 20159 


\section{C. Weber et al.}

This paper has been typeset from a $\mathrm{T}_{\mathrm{E}} \mathrm{X} / \mathrm{LT}_{\mathrm{E}} \mathrm{X}$ file prepared by the author. 